\title{
Sistemas multiagentes: mapeando a evasão na educação a distância
}

\author{
Beatriz Wilges, UFSC/(PGEGC), beaw@inf.ufsc.br \\ Júlio César da Costa Ribas, UFSC/(PGEGC)/IF-SC, julio@ifsc.edu.br \\ Araci Hack Catapan, UFSC/(PGEGC), aracihack@gmail.com \\ Rogério Cid Bastos, UFSC/(PGEGC), rogerio@inf.ufsc.br
}

\begin{abstract}
Resumo: Uma instituição, ao adotar à modalidade a distância, enfrenta preocupações que envolvem principalmente o fantasma da evasão. Esse fantasma não é prerrogativa de uma instituição em particular, mas de todas as instituições públicas e privadas. Estudar as causas que contribuem para a permanência e êxito escolar é combater a evasão. Para isso, é preciso trabalhar com duas frentes: uma de ação imediata, que busca resgatar o aluno "evadido", e outra de reestruturação interna, que implica a discussão e avaliação não só de combate à evasão, mas ao fracasso escolar como um todo. $\mathrm{O}$ artigo apresenta uma proposta de modelo conceitual preditivo de evasão na modalidade de educação a distância, modelado sob uma arquitetura de Sistema Multiagentes. Espera-se que, a partir desta proposição, o problema da evasão venha a ser mais bem visualizado, auferindo novas perspectivas de gestão.
\end{abstract}

Palavras-chave: evasão, EaD, modelagem de SMA.

\section{Multiagent systems: mapping evasion in distance education}

Abstract: When an institution adopts a distance teaching modality, it faces concerns that involve mainly the risk of evasion. This risk is not related to one particular institution, but on the contrary it is linked to all public and private institutions. Studying the causes that contribute to the academic success and persistence is to fight against evasion. In order to accomplish this, it is necessary to work in two ways: one way that takes immediate actions to rescue the "evaded" student, and another way that involves the internal restructuring of distance teaching courses. This implies an evaluation and discussion not only to fight evasion, but also to fight school failure as a whole. The article proposes a conceptual model for predicting dropout in distance teaching mode modeled in a Multiagent System Architecture. We hope that this will help the problem of evasion to be better understood, and new management perspectives are achieved.

Keywords: evasion, distance education, MAS modeling.

\section{A EDUCAÇÃO A DISTÂNCIA NO CENÁRIO NACIONAL E REGIONAL}

Segundo Belloni (1999), uma das grandes dificuldades de EaD tem que ver com sua posição de baixo prestígio no campo da educação. Tendo sido considerada por longo tempo como uma solução paliativa, emergencial ou marginal com relação aos sistemas convencionais, a EaD é geralmente vista como uma segunda oportunidade para os que não tiveram acesso ou abandonaram o ensino regular. Esta percepção gera dúvidas quanto à qualidade do ensino oferecido por sistemas de $\mathrm{EaD}$ e tende a enfatizar os fracassos, não obstante o sucesso de algumas das grandes universidades abertas européias (Belloni, 1999).

De acordo com Schneider (1999), as universidades européias a distância têm incorporado em seu desenvolvimento histórico as novas tecnologias de informática e de telecomunicações. Um exemplo disso é o desenvolvimento da Universidade a Distância de Hagen, que iniciou seu programa com material escrito em 1975 e hoje oferece 
material didático em áudio e videocassetes, videotexto interativo e videoconferências. Tendências similares podem ser observadas nas Universidades Abertas da Inglaterra, da Holanda e da Espanha. Hoje mais de 80 países, nos cinco continentes, adotam a EaD em todos os níveis de ensino, em programas formais e não formais, atendendo a milhões de estudantes (Schneider, 1999).

Nesse contexto de quebra de paradigmas culturais e de concretização de um modelo de EaD pertinente, viável e de qualidade no Brasil, a exemplo do cenário mundial, o Ministério da Educação, por meio de sua Secretaria de Educação a Distância - SEED, buscando incentivar e coordenar projetos para estruturar a EaD nacional, criou em 2006 o Sistema Universidade Aberta do Brasil - UAB.

Na região Sul, a EaD está presente em várias instituições de ensino e ganha gradativa importância em cursos de qualificação profissional que contam com o apoio da iniciativa privada. De acordo com o Anuário Brasileiro Estatístico de Educação Aberta e a Distância de 2008, a região Sul está em segundo lugar no cenário brasileiro, com $27 \%$ de instituições autorizadas oficialmente para ofertar cursos de EaD, atrás somente da região Sudeste, que apresenta um percentual 39,2\%.

\section{A EVASÃO ESCOLAR E A MODALIDADE DE ENSINO A DISTÂNCIA}

Conhecer a EaD implica buscar na historicidade dos atos e fatos a compreensão das possibilidades de transformações, das dificuldades e facilidades. Significa inserir-se e integrar-se num tempo e espaço, num contexto de demandas por formação e suas influências sobre a educação presencial que conhecemos e a não-presencial, na reorganização do trabalho docente e nos processos de aprendizagem (Martins, 2007).

Historicamente, são semelhantes as preocupações na educação presencial e na educação a distância: as duas buscam novas formas de acesso ao conhecimento que possibilitem a construção de conceitos, uma maior compreensão das idéias e do pensamento analítico e crítico, mediante o uso de recursos tecnológicos.

Inicialmente, a EaD se consolidou como ensino por correspondência, cujos materiais eram distribuídos por sistema de postagem. Mais tarde, o livro impresso representou o marco inicial para a disseminação da palavra escrita. Posteriormente, a introdução de novos meios de comunicação de massa, como o rádio, disponível desde o início do século $\mathrm{XX}$, originou projetos importantes, principalmente no meio rural, permitindo que o som e a voz pudessem ser levados a localidades distantes. As várias ações desenvolvidas nas instituições, no campo da educação secundária e superior, a partir das décadas de 60 e 70, caracterizaram um grande avanço nessa modalidade de educação, começando pela Europa (França e Inglaterra) e se expandindo pelos demais continentes.

Concluímos, no tocante à contextualização histórica da Educação a Distância no cenário mundial, que a mesma é utilizada em muitos países em todos os níveis de ensino, em sistemas formais e não-formais, atendendo a milhões de estudantes. As estatísticas apontam para o crescente número de empresas que capacitam recursos humanos através da EaD, em especial na Europa e nos Estados Unidos. Os recursos utilizados mudam com a evolução tecnológica. Por outro lado, não são excludentes, pois agregam novas possibilidades para atender as exigências da contemporaneidade.

Acompanhando a evolução histórica da EaD no cenário mundial, o crescimento da Educação a Distância no Brasil vem apresentando números expressivos. Os números oferecidos pelo INEP/MEC comprovam a permanente expansão do sistema. De 2003 a 2008, o número de cursos de graduação passou de 52 para 466, o que representa aumento de $796 \%$. O crescimento nesses cursos também superou expectativas. As 
matrículas passaram de 49 mil em 2003 para 760 mil em 2008, numa elevação de 1451\% (AbraEaD, 2008), (CensoEAD.BR, 2010).

Se, por um lado, a oferta de EaD cresce vigorosamente, a investigação sobre a evasão escolar ganha um capítulo de destaque. A questão é de grande importância, pois é também necessário conhecer os fatores que podem levar um estudante a abandonar um curso. Embora cada vez mais estudos se dediquem a esta matéria, ainda não há um mapeamento completo em todos os seus recortes, até porque a própria estrutura formal da EaD ainda não encontrou assentos legais e institucionais estáveis.

A evasão é definida como a saída do estudante de um curso ou do sistema de educação sem concluí-lo com sucesso. Segundo o relatório da Comissão Especial de Estudo sobre Evasão nas Universidades Públicas Brasileiras, apresentado em 1996, existem três tipos de evasão (Brasil, 1996):

1. evasão de curso - quando o estudante desliga-se do curso em situações diversas, tais como: abandono, desistência, transferência interna ou externa, exclusão por regimento institucional;

2. evasão da instituição - quando o estudante desliga-se da instituição na qual está matriculado;

3. evasão do sistema - quando o estudante abandona de forma definitiva ou temporária o ensino superior.

Para Favero (2006), considera-se evasão a desistência do curso, incluindo os que, após se terem matriculado, nunca se apresentaram ou se manifestaram de alguma forma para os colegas e mediadores do curso, em qualquer momento. Evasão escolar é, portanto, uma interrupção de um ciclo de estudo, quando o estudante deixa de completar o curso ou programa que frequenta. Esta é uma realidade não só em nosso país, mas também em outros países.

Em cursos a distância, a evasão é tida como um fator frequente. O êxito em um curso pode ter como influência diversos fatores, entre eles: uma definição clara do programa, a utilização correta do material didático, o uso correto de meios apropriados que facilitem a interatividade entre professores e alunos e entre os alunos, e a capacitação dos professores. Afora esses aspectos, a evasão pode também ser influenciada por razões profissionais, dificuldades acadêmicas, motivos familiares, motivos pessoais, entre outros.

Segundo o Relatório Analítico da Aprendizagem a Distância no Brasil (CensoEAD.BR, 2010), a evasão média apurada entre as instituições pesquisadas é de $18,5 \%$, com variantes significativas em alguns recortes. No setor público, ela é de $21,1 \%$ e no setor privado é de $17,3 \%$. Os maiores índices médios de evasão no País estão no Norte $(27,8 \%)$ e Nordeste $(21,7 \%)$. Os menores no Sul $(14,8 \%)$, Sudeste $(19,0 \%)$ e Centro-Oeste $(17,3 \%)$.

De acordo com Martinez apud Comarella (2009),

na EaD os tipos de evasão são classificados da seguinte forma: Dropout (evasão) ocorre quando o estudante abandona o curso ou o sistema de educação durante o seu desenvolvimento e nunca retorna. Já o stopout (trancamento) é a interrupção temporária do curso, e attainer (evasão do curso) ocorre quando o estudante sai do curso antes da sua conclusão, mas com a aquisição do conhecimento, ou por ter atingido suas metas pessoais. Já o caso dos estudantes que nem chegam a iniciar o curso é chamado de non-starter (não iniciante). $\mathrm{O}$ conceito de evasão é equivalente tanto nos cursos presenciais como 
nos cursos a distância: é o desligamento do estudante do curso ou da instituição de ensino superior (IES) na qual está matriculado.

Segundo Levy (2007), há três conjuntos de variáveis responsáveis pela evasão: (1) o lócus de controle do acadêmico, ou seja, se ele se considera responsável ou não por sua situação. Se o estudante tiver um lócus de controle interno significa que ele acredita que o seu sucesso depende do seu esforço pessoal; se ele tem um lócus de controle externo, ele atribui a responsabilidade do seu sucesso a outras pessoas, sorte, chance, etc.; (2) a satisfação dos estudantes com a aprendizagem em cursos online; e (3) a influência das características demográficas (idade, sexo, localização geográfica, tipo de diplomas, entre outras) dos estudantes.

Para combater a evasão escolar, portanto, é preciso trabalhar com duas frentes: uma de ação imediata que busca resgatar o aluno "evadido", e outra de reestruturação interna que implica a discussão e avaliação não só de combate à evasão, mas ao fracasso escolar como um todo. Isso implica dizer que algo precisa ser feito para garantir a permanência do aluno no seu itinerário de formação, em especial o aluno a distância, que estatisticamente possui índice de evasão significativo, da ordem de 30\% (Favero, 2006).

É sob esse enfoque e sobre a importância de gerar recursos que auxiliem os gestores institucionais e professores a evitar a evasão escolar que este trabalho foi gerado. Acredita-se que a utilização de um Sistema Multiagentes dentro de um Ambiente Virtual de Aprendizagem (AVEA) pode contribuir no sentido de gerar um índice que, avaliado sob uma escala, pode indicar o quanto um estudante está próximo de uma situação de evasão.

\section{SISTEMA MULTIAGENTES}

A proposta desse trabalho envolve a modelagem de uma comunidade de agentes que possa ajudar a baixar os altos índices de evasão escolar. Dentro dessa perspectiva, espera-se que a implementação de um Sistema Multiagentes (SMA) possa fornecer dados significativos e relevantes para recuperar um possível estudante desistente antes que a evasão se concretize. Para entender o funcionamento de um Sistema Multiagentes, inicia-se esta seção resgatando referências na literatura sobre o que são agentes, bem como um Sistema Multiagentes.

De acordo com Bradshaw (1997), um agente é descrito como uma entidade de software que funciona de forma contínua e autônoma em um ambiente em particular, geralmente habitado por outros agentes, e que seja capaz de intervir no seu ambiente, de forma flexível e inteligente, sem requerer intervenção ou orientação humana constante. De um modo ideal, um agente que funcione continuamente, por longos períodos de tempo, deve ser capaz de aprender com a experiência e, se ele habita um ambiente com outros agentes, deve ser capaz de comunicar-se e cooperar com eles, e ainda mover-se de um local para outro.

Um segundo conceito chave, dentro dessa proposta, seria a estratégia adotada por estes agentes. A estratégia envolve a maneira como um agente reage ao seu meio e persegue seus objetivos. Um dos grandes interesses dessa proposta é como as estratégias mudam ao longo do tempo. Uma fonte de mudança é a experiência do agente em quão bem a estratégia está se saindo. Por esse lado, podemos observar as questões de adaptação vinculadas às estratégias de um determinado agente. Isso ocorre no intuito de melhorar o processo no qual o agente está envolvido, a fim de alcançar com maior eficiência seus objetivos e resultados esperados. 
A idéia de uma população de agentes surge com o objetivo de aplicar, na proposta desta pesquisa, uma abordagem que contemple os mais variados e importantes perfis de agentes, com comportamentos e objetivos bem definidos, para que o modelo de SMA proposto possa estar em contínuo processo de aprendizagem.

Os avanços mais recentes no campo dos ambientes de aprendizagem inteligentes têm proposto o uso de arquiteturas baseadas em sociedades de agentes. Os princípios dos Sistemas Multiagentes (SMA) têm mostrado um potencial bastante adequado ao desenvolvimento de sistemas de ensino-aprendizagem, devido ao fato de que, pela própria natureza dos problemas de ensino-aprendizagem, estes são mais facilmente resolvidos de forma cooperativa (Silveira, 2000).

O uso de um SMA é justificado quando a resolução de um objetivo requer o esforço de dois ou mais agentes, e isso é o que denominamos de Sistema Multiagentes. Além disso, um SMA pode ser definido como uma rede de resolvedores de problema que trabalham juntos para solucionar problemas que estão além das capacidades individuais (ou conhecimentos individuais) de cada resolvedor de problema (Jennings, 1998).

De acordo com Silveira, Gomes e Vicari (2003), um sistema computacional de ensino que procure solucionar o problema de ensino/aprendizagem em ambientes de EAD deve contemplar aspectos tanto de IA quanto de Redes de Computadores. Baseados nesse princípio, começaram a surgir os chamados Ambientes Inteligentes Distribuídos de Aprendizagem (DILE - Distributed Intelligent Learning Environment). O ambiente JADE, Java Agent Framework for Distance Learning Environment, propõe um conjunto de recursos para a construção de DILE. Ele utiliza a WWW e é baseado numa arquitetura multiagentes. Portanto, a maior interatividade e adaptação ao estudante são buscadas através da interação e da cooperação entre os seus agentes.

Além do ambiente JADE, dispõe-se também da organização FIPA (Foundation for Intelligent Physical Agents). A FIPA surgiu como um esforço na tentativa de padronizar as tecnologias de comunicação entre agentes, que, até o seu surgimento, eram completamente heterogêneas. Para tal, ela desenvolveu um conjunto de documentos, entre os quais está a descrição de uma linguagem de comunicação totalmente voltada para a comunicação entre agentes: a FIPA-ACL (2010). Os padrões FIPA vêm crescendo em uso e qualidade, o que pode ser verificado através da observação da grande variedade disponível de plataformas de desenvolvimento de agentes compatíveis com eles.

Nesse trabalho, será utilizado o AgentTool (2010) para especificar o modelo proposto. A implementação e validação dessa proposta dentro de um ambiente como o FIPA e o JADE está sendo estudada e pesquisada e não faz parte do escopo deste trabalho.

\section{O MODELO CONCEITUAL PREDITIVO DE EVASÃO}

A tarefa de projetar um modelo de SMA que, a partir de qualquer Ambiente Virtual de Ensino-Aprendizagem (AVEA), possa extrair informações sobre previsões de comportamento não é uma atividade trivial. Esse tipo de modelagem requer uma análise detalhada sobre as tarefas e objetivos envolvidos no SMA. Sugere-se, neste trabalho, que qualquer AVEA implemente as mesmas características, no intuito de evitar ao máximo os altos índices de evasão.

Para facilitar a análise e identificação dos principais agentes envolvidos na arquitetura do SMA proposto, utilizamos uma ferramenta denominada AgentTool (2010). O AgentTool é um ambiente de desenvolvimento gráfico implementado em Java para ajudar os usuários a analisar, projetar e implementar SMA. Ele é projetado 
para apoiar a Metodologia de Engenharia de Sistemas Multiagentes (Multiagent Systems Engineering - MaSE).

Para especificar a troca de mensagens entre os papéis envolvidos no modelo de SMA proposto, a ferramenta AgentTool utiliza a especificação de casos de uso, que são expressos também por diagramas de sequência. Diagrama de sequência (ou Diagrama de Sequência de Mensagens) é um diagrama usado em UML (Unified Modeling Language), representando a sequência de processos (mais especificamente, de mensagens passadas entre objetos) num programa de computador. Este diagrama é construído a partir do Diagrama de Casos de Usos. Primeiro, se define qual o papel do sistema (Use Cases), depois, é definido como o software realizará seu papel (Sequência de operações).

Na Figura 1, é apresentada a hierarquia de objetivos do SMA proposta conforme a metodologia da ferramenta AgentTool.

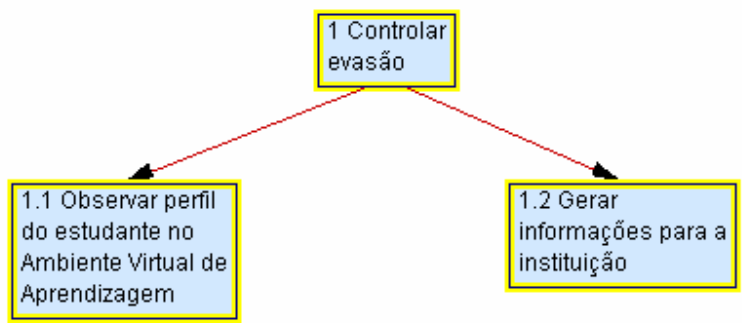

Figura 1. Hierarquia dos objetivos do SMA proposto.

A partir da definição da hierarquia dos objetivos propostos, construímos o diagrama do modelo de agentes envolvidos na arquitetura do Sistema Multiagentes. A Figura 2 apresenta esse diagrama.

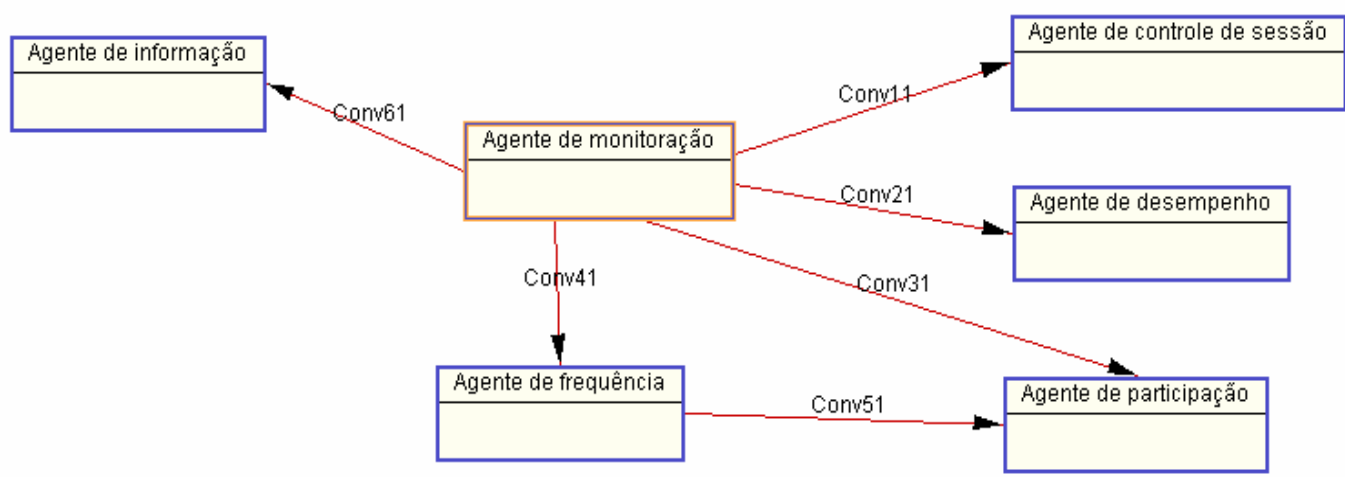

Figura 2. Diagrama do modelo de agentes

A definição dos agentes envolvidos no diagrama (Figura 2) baseou-se nas características gerais dos atuais Ambientes Virtuais de Ensino-Aprendizagem (AVEA). Isso significa que qualquer AVEA deve implementar um agente para controlar a sessão de aprendizagem dos estudantes, um agente para monitorar o desempenho, um agente para controlar a participação em wikis e fóruns, um agente para observar a frequência do estudante e um agente central, que é o agente de monitoração. Este agente está em constante comunicação com os demais para, em caso de observação de risco de evasão, comunicar o agente de informação. O agente de informação é o responsável por avisar o professor, bem como a instituição. Os diagramas de sequência de cada um desses 
agentes são apresentados a seguir. O primeiro diagrama é relativo ao agente que controla a sessão, ou seja, o rastreamento dos logs (Figura 3).

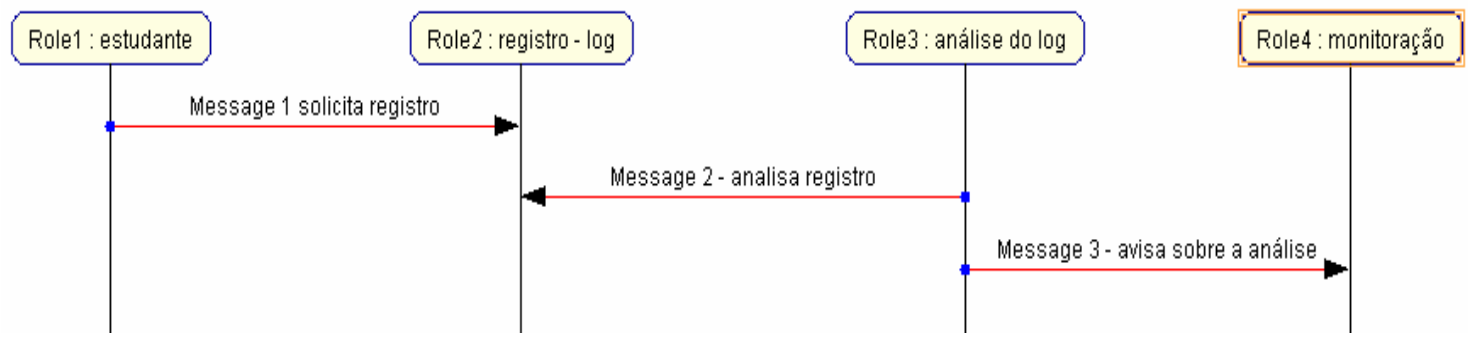

Fig. 3. Diagrama de sequência do agente de controle da sessão

No diagrama acima, é apresentado o papel do estudante navegando no AVEA e na sequência os registros sendo armazenados em um BD. Sob esses registros existe (papel 3) a análise dos mesmos, que repassa as informações pertinentes ao agente de controle. Na Figura 4, é apresentado o diagrama de sequência do agente de desempenho.

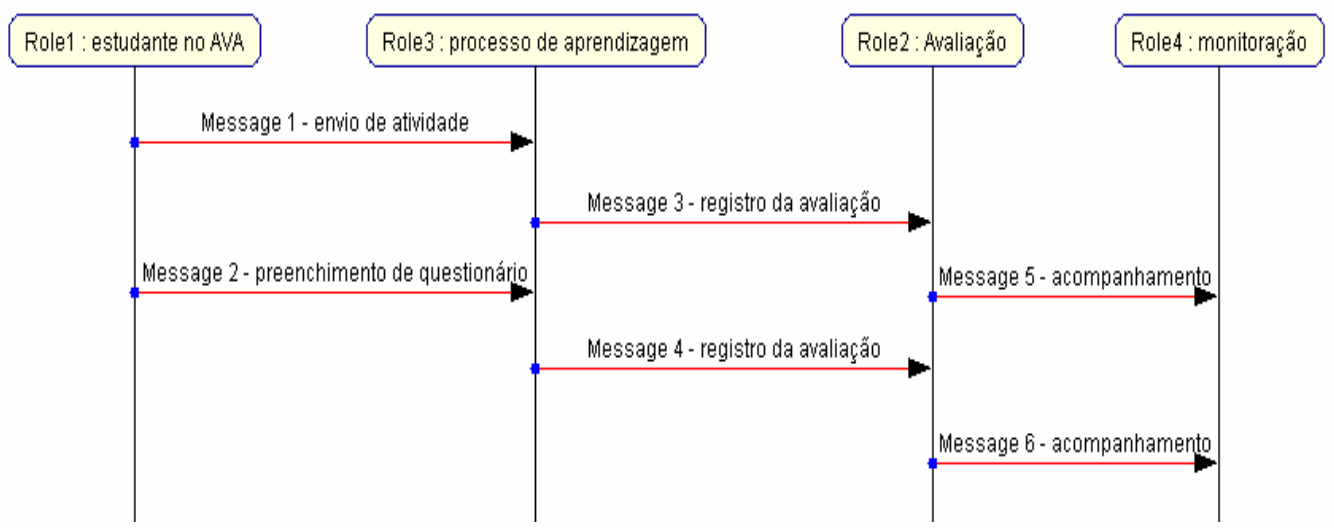

Figura 4. Diagrama de sequência do agente de desempenho

Nesse diagrama, é mostrado o funcionamento da análise de desempenho dos estudantes. O estudante em processo de aprendizagem está em constante avaliação. Os registros das avaliações são submetidos a um quarto papel, que é representado pelo agente de monitoração. Na Figura 5, é mostrado o diagrama de sequência do caso de uso que envolve o agente que analisa a frequência.

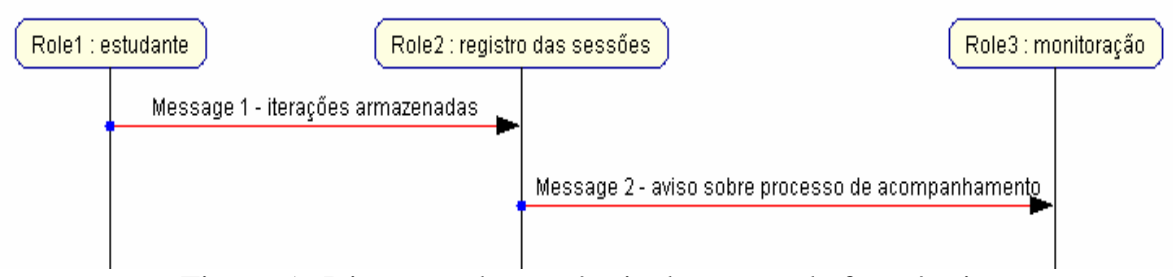

Figura 5. Diagrama de sequência do agente de freqüência

Na Figura 5, percebe-se o movimento de acompanhamento da aprendizagem. Nesse momento, verifica-se o tempo e frequência de acesso nas sessões do AVEA. Tudo é registrado para posteriormente informar o agente de monitoração. E, por fim, tem-se o diagrama de sequência do agente participação (Figura 6). 


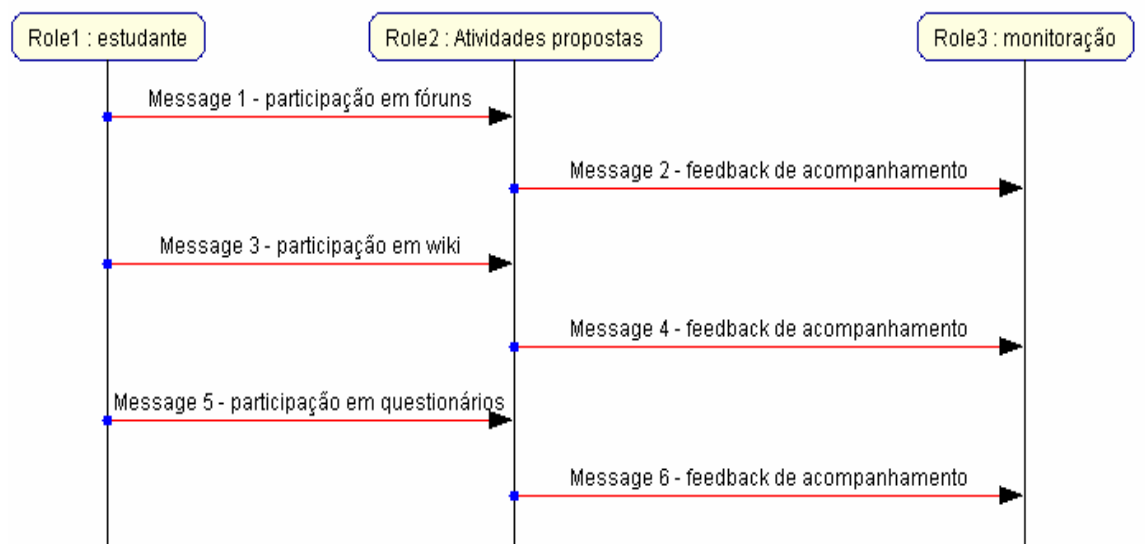

Figura 6. Diagrama de sequência do agente de participação

Na Figura 6, são exemplificadas as atividades que são observadas para caracterizar a participação dos estudantes. São consideradas atividades propostas em qualquer AVEA os fóruns, wikis e os questionários. A partir dessas atividades, é possível avaliar o grau de interação dos estudantes e informar ao agente de monitoração.

Todos esses diagramas mostram os procedimentos envolvidos para que, a partir da coleta dessas informações, o agente de controle calcule o risco de evasão envolvido. Esse monitoramento e avaliação são constantes e dinâmicos para permitir um resultado mais preciso sobre a análise do risco envolvido.

\section{CONSIDERAÇÕES FINAIS}

A origem da abordagem sistêmica reside na Teoria Geral de Sistemas (TGS). Assim como nessa proposta, a abordagem sistêmica observa um problema de maneira holística, como um todo, sendo este formado pelas relações, objetivos, elementos e o próprio meio em que está inserido. Normalmente, essa abordagem contribui para encontrar-se uma solução, quase sempre adequada. Segundo Alves [18], "a abordagem sistêmica, através da TGS, oferece essa alternativa, descobrindo princípios comuns a todo tipo de sistema, o que facilita estudos de cada um deles".

Estima-se que essa proposta consolide uma série de fatores que são relevantes para avaliação do risco de evasão. Considera-se, a partir de todas as referências utilizadas nessa pesquisa, que este trabalho tem uma ampla abordagem de pesquisa, envolvendo todo o âmbito do atual processo de educação a distância. O objetivo dessa comunidade de agentes, conforme as características descritas nesse trabalho é possibilitar o retorno de resultados mais satisfatórios para qualquer Ambiente Virtual de Ensino-Aprendizagem (AVEA), de forma que haja um acompanhamento e monitoramento dos estudantes em todas as atividades que envolvem o ensino a distância.

Uma proposta de trabalho futuro nessa perspectiva é implementar essa comunidade de agentes do modelo proposto em uma AVEA e avaliar o quanto esse modelo de Sistema Multiagentes (SMA) pode contribuir para redução da evasão e até mesmo promover um maior número de estudantes dentro das instituições de ensino na modalidade à distância.

\section{REFERÊNCIAS BIBLIOGRÁFICAS}

ABRAEAD. Anuário Brasileiro Estatístico de Educação Aberta e a Distância. AbraEAD2008. ABED. Instituto Cultural e Editorial Monitor São Paulo. 2008. 
ALVES, J. B. M. Material da disciplina de Teoria Geral de Sistemas. Disponível em http://www.inf.ufsc.br/ jbosco/tgs/tgs2005-1.html [Material didático; Notas de aula]. acessado em 15 mai. 2010.

AGENTETOOL. Disponível em:

http://macr.cis.ksu.edu/projects/agentTool/agentool.htm Acesso em: abril de 2010.

BELLONI, Maria Luiza. Educação a distância. Campinas: Autores Associados, 1999. p. 91.

BRADSHAW, J. M. An introduction to software agents In: BRADSHAW, J. M. Ed. Software Agents. Massachusetts: MIT Press, 1997.

BRASIL, Ministério da Educação Superior. Diplomação, retenção e evasão nos cursos de graduação em instituições de ensino superior públicas. Relatório da Comissão Especial de Estudos nas Universidades Públicas Brasileiras.

ANDIFES/ABRUEM/SESu/MEC: 1996.

CENSOEAD.BR. Relatório analítico da aprendizagem a distância no Brasil. São Paulo: Pearson Education do Brasil, 2010.

COMARELLA, Rafaela Lunardi. Educação superior a distância: evasão discente. 2009. 147 p. Dissertação (Mestrado) - Programa de Pós-Graduação em Engenharia e Gestão do Conhecimento, Universidade Federal de Santa Catarina, Florianópolis, 2009.

FAVERO, Rute Vera Maria. Dialogar ou evadir: Eis a questão!: Um estudo sobre a permanência e a evasão na Educação a Distância, no Estado do Rio Grande do Sul. 2006. 169 f. Dissertação (Mestrado) - Universidade Federal do Rio Grande do Sul, Porto Alegre, 2006.

FIPA Specifications Repository. Disponível em:

http://www.fipa.org/specs/pesspecs.tar.gz Acesso em: abril de 2010.

JENNINGS, Nicholas R. et al. A Roadmap of Agent Research and Development. Autonomous Agents and Multi-Agent Systems. Kluwer Academic Publishers, Boston, 1, p. 275-306,1998.

LANDIM, Claudia Maria das Mercês Paes Ferreira. Educação a distância: algumas considerações. Niterói: [s. n.], 1997.

LEVY, Y. Comparing dropouts and persistence in e-learning courses. Computers \& Education, v.48, p.185-204, 2007.

MARTINS, Janae Gonçalves; BEBER, Bernadétte. Produção de Material Didático Impresso para EaD. Anais do $13^{\circ}$ Congresso Internacional de Educação a Distância. Curitiba. 2007.

SCHNEIDER, Maria Clara Kaschny. Educação a distância: desafios para a interação na sala de aula virtual pautados na transposição da tecnologia nos projetos de videoconferência. 1999. Dissertação (Mestrado em Engenharia de Produção) Programa de Pós-Graduação em Engenharia de Produção. Universidade Federal de Santa Catarina, Florianópolis, 1999.

SILVEIRA, Ricardo Azambuja. Modelagem orientada a agentes aplicada a ambientes inteligentes distribuídos de ensino - JADE - Java Agent framework for Distance learning Environments. 2000. 126f. Tese (Doutorado em Ciência em Educação) Instituto de Informática. Universidade Federal do Rio Grande do Sul, Porto Alegre, 2000. 
SILVEIRA, R. A.; Gomes, E. R.; Vicari, R. M. Modelagem de Ambientes de Aprendizagem baseado na Utilização de Agentes FIPA. Proceedings of Simpósio Brasileiro de Informática na Educação 2003. Rio de Janeiro, 2003.

RIBEIRO, C. A. Desenvolvimento Curricular. Lisboa: Texto Editora, 1990. 